\title{
Impact of Formulation Conditions on Lipid Nanoparticle Characteristics and Functional Delivery of CRISPR RNP for Gene Knock-Out and Correction
}

\author{
Johanna Walther't', Danny Wilbie ${ }^{1}+$, Vincent S.J. Tissingh ${ }^{1}$, Mert Öktem ${ }^{1}$, Heleen van der Veen ${ }^{1}$, Bo Lou ${ }^{1}$, Enrico \\ Mastrobattista $^{1^{*}}$ \\ 1 Department of Pharmaceutics, Utrecht Institute of Pharmaceutical Sciences (UIPS) , Utrecht University , \\ Universiteitsweg 99 , 3584 CG Utrecht, The Netherlands. \\ + These authors contributed equally to this work. \\ * Corresponding author, email address: e.mastrobattista@uu.nl
}

\begin{abstract}
The CRISPR-Cas9 system is an emerging therapeutic tool with the potential to correct diverse genetic disorders. However, for gene therapy applications an efficient delivery vehicle is required, capable of delivering the CRISPR-Cas9 components into the cytosol of the intended target cell population. Once there, the ribonucleoprotein complex (RNP) can be transported into the nucleus. Lipid nanoparticles (LNP) serve as promising candidates for delivery of CRISPR-Cas9 RNP. These delivery vehicles have been optimized for the delivery of nucleic acids, such as mRNA. Codelivery of Cas9 encoding mRNA with the accompanying sgRNA leads to translation of the Cas9 protein and formation of the Cas9 RNP inside the cell. Only recently, direct delivery of the CRISPRCas9 RNP complexes has been explored, which requires adjustments to the LNP formulation. In this study, the importance of buffer composition and cationic charge during RNP and ssDNA entrapment in LNP are demonstrated. After optimizing several formulation parameters, LNP were prepared that were colloidally stable in human plasma and efficiently deliver the SpCas9 RNP and ssDNA for HDR-correction in reporter cells. Under optimal formulation conditions, gene knock-out and gene correction efficiencies as high as $80 \%$ and $20 \%$, respectively were achieved at nanomolar CRISPR-Cas9 RNP concentrations.
\end{abstract}

Keywords: CRISPR, LNP, formulation, NHEJ, HDR, AF4, delivery

\section{Introduction}

The Clustered Regularly Interspaced Short Palindromic Repeats (CRISPR) associated (Cas) endonuclease proteins, such as Cas9, have emerged in recent years as a viable therapeutic option for genetic diseases. The Cas 9 endonuclease was first identified as a bacterial defence mechanism against viral infections, and has been repurposed into a powerful tool to cleave DNA in an RNA-guided fashion in various cell types. The Cas9 protein, together with a guide RNA (gRNA) molecule, forms an active ribonucleoprotein (RNP) complex [1]. DNA cleavage is mediated by recognition of a 20 nucleotide sequence between the gRNA and the host DNA, which hybridize and allow the nuclease to attach to its DNA target. Additionally, the presence of a protospacer-adjacent motif (PAM) in the host DNA is necessary to facilitate the conformational change in the nuclease to introduce a double strand break (DSB) in its target [2]. When the genomic DNA is cleaved by the Cas9 enzyme the host DNA-damage repair response is activated [3]. In mammalian cells the most prominent pathways are the canonical non-homologous end-joining (c-NHEJ) pathway, the microhomology-mediated end joining (MMEJ) pathway and homology-directed repair (HDR) [4]. C-NHEJ and MMEJ are notably error-prone repair mechanisms, both of which can lead to formation of small insertions and deletions in the target gene. This in turn may lead to gene knock-out, which is therapeutically relevant for gene therapy of diseases caused by gain-of-function mutations [5-7]. HDR is mostly active in the G2/S phases of mitosis in dividing cells, and in the presence of a homologous DNA 
template this pathway can lead to precise DNA repair of disrupted genes [8]. Especially the latter signifies potential for gene therapy: curing diseases by editing and correcting the genetic mutations.

Direct in vivo gene editing requires the delivery of the CRISPR-Cas9 components into the correct target cells' nuclei [9]. SpCas9, a Cas9 protein derived from Streptococcus pyogenes, is currently under clinical investigation for both ex-vivo and direct in vivo therapeutic application [10-12]. Examples include subretinal injection of adeno-associated viral vectors encoding the CRISPR-Cas9 components for the treatment of Leber congenital amaurosis, and delivery of CRISPR-Cas9 with non-viral particles such as NTLA-2001 for targeted gene editing of hepatocytes for hereditary amyloid transthyretin amyloidosis $[13,14]$. Lipid nanoparticles (LNP), which employ cationic or ionizable cationic lipids, serve as particularly promising candidates for delivery of the different cargo formats of the CRISPR-Cas9 components. Since LNP complex their cargo via electrostatic interactions, they are especially suited to formulate polyanionic DNA or RNA molecules, due to their anionic phosphate backbone. However, the preassembled RNP complex, with or without co-entrapment of a DNA template to drive homology-directed repair, can also be formulated in LNPs as was recently demonstrated [13,15-17].

Direct delivery of the pre-assembled RNP has several advantages over Cas9 expressed from DNA or mRNA templates. Since RNP are pre-assembled they are directly active once inside the nucleus of target cells as opposed to Cas9 expression from DNA or mRNA templates. These need to first be translated into the endonuclease in the cytosol, and subsequently find an intact single guide RNA (sgRNA) within the cell in order to become active [18]. Related to this, direct delivery of RNP assures optimal stoichiometry between Cas9 and sgRNA and protects the sgRNA from rapid degradation within the cell [19]. Finally, RNP are short lived inside cells, with a half-life of approximately one day [20]. This limits the likelihood of off-target gene editing which has been shown to be time dependent $[21,22]$.

Despite these advantages, delivery of RNP has met with several pharmaceutical challenges. The stability of RNP during LNP formulation is an issue. Solely relying on ionizable cationic lipids to mediate electrostatic interactions with net-negative charged RNP requires an acidic environment. Acidic conditions can however affect RNP stability [23,24]. Formulation conditions must be optimized to find a good balance between RNP functionality, protection from premature clearance and timely intracellular release. This work sought to explore several of such often overlooked steps in the pharmaceutical formulation of RNP into LNP, which, as shown here, are often critical in determining gene editing efficiency [23]. This includes buffer composition during formulation, as well as lipid composition of LNP for delivering RNP with or without a single stranded DNA (ssDNA) HDR templates. To understand the effects of these parameters, these LNP were characterized on their size, surface charge, RNP complexation, and activity. Additionally, their stability in human plasma was studied. Lipid nanoparticles complexing RNP and HDR template were investigated on gene editing capacity in fluorescent reporter cell lines suited to read out gene knock-out and specific gene correction, resulting in promising results for in vivo gene correction. 


\section{Materials and Methods}

\section{General reagents}

All reagents and chemicals were acquired from Sigma-Aldrich (Zwijndrecht, the Netherlands) unless otherwise specified. 2' O-methyl and phosphorothioate end-modified sgRNA and template DNA sequences were acquired from Sigma-Aldrich (Haverhill, the United Kingdom, sequences given in S.1) and stored in RNAse-free Tris EDTA-buffer pH 7.0 (Thermo Fisher, Landsmeer, the Netherlands). PCR primers were acquired from Integrated DNA Technologies (IDT, Leuven, Belgium). C12-200 lipid [25] was acquired from CordonPharma (Plankstadt, Germany), DOPE from Lipoid (Steinhausen, Switzerland), Cholesterol and PEG-DMG from Sigma-Aldrich (Zwijndrecht, the Netherlands) and DOTAP from Merck (Darmstadt, Germany).

\section{SpCas9 Protein Production and Purification}

SpCas9 with a nuclear localization signal (NLS) was expressed in the LPS-free Clearcoli $^{\mathrm{TM}}$ BL21 strain (Lucigen Corporation, Wisconsin USA) using the pET15_SpCas9_NLS_His plasmid (Addgene \#62731) [26]. After growth in LB-Miller medium until the OD600 reached $0.55-0.7$, protein production was induced with $0.5 \mathrm{mM}$ isopropyl $\beta$-D-1-thiogalactopyranoside (IPTG) followed by overnight fermentation at $18{ }^{\circ} \mathrm{C}$. All bacteria were subsequently pelleted by centrifugation and lysed by tip sonication using a 3mm tip (Bandelin electronic GmbH \& Co. KG, Berlin, Germany), in $50 \mathrm{ml}$ of PBS containing $25 \mathrm{mM}$ imidazole on ice. The lysate was subsequently centrifuged, resuspended in the same buffer, and filtered through a $0.45 \mu \mathrm{m}$ MiniSart filter (Sartorius, Amersfoort, the Netherlands). Immobilized metal affinity chromatography (IMAC) was performed on this lysate using a $1 \mathrm{ml}$ nickel HisTrap HP column (Cytiva, Medemblik, the Netherlands) in combination with the Äkta PURE chromatography system (Cytiva, Medemblik, the Netherlands). A stepwise gradient of imidazole was applied from $25 \mathrm{mM}$, going up to $100 \mathrm{mM}$ and ending at $250 \mathrm{mM}$.

After collection of all fractions, the eluted SpCas9 was dialyzed twice against storage buffer (final composition of $300 \mathrm{mM} \mathrm{NaCl}, 0.1 \mathrm{mM}$ EDTA, $10 \mathrm{mM}$ Tris, pH 7.4) at a 1:1000 ratio of sample to dialysate, followed by addition of $8.3 \%(\mathrm{w} / \mathrm{v})$ glycerol prior to freezing. The samples were snap-frozen in liquid nitrogen and stored at $-80{ }^{\circ} \mathrm{C}$ after dialysis.

\section{SpCas9 characterization and stability study}

The protein size and protein impurities were assessed using sodium dodecyl sulfate polyacrylamide gel electrophoresis (SDS-PAGE). The samples were treated with Laemmli sample buffer containing $12.5 \mathrm{mM}$ dithiothreitol (DTT). The proteins were separated on a 4-12\% Bis-Tris gel (Thermo Fisher, Landsmeer, the Netherlands), after which staining was done using the Pierce silver stain kit (Fischer Scientific, Landsmeer, the Netherlands). Gels were imaged in the ChemiDoc Imaging System (Bio-Rad Laboratories B.V, Veenendaal, the Netherlands). The intensity of the gel bands was quantified by densitometry in ImageJ (version 1.52p), to calculate the protein impurities in the SpCas9 samples over time [27]. This assay was repeated periodically to determine the protein stability during 6 months of storage.

To visualize in-vitro cleaving activity of SpCas9, an in-house optimized activity assay was performed. SpCas9 was first incubated with sgRNA specific for the EGFP gene (Supplementary S.1) for 10 minutes at room temperature, at a molar ratio of 1:1 at a concentration of $1 \mu \mathrm{M}$. Subsequently, $2 \mu \mathrm{l}$ of this RNP was mixed with $3 \mu \mathrm{l}$ Buffer 3.1 10X, (New England Biolabs, Ipswich, USA), 250 ng linearized plasmid DNA containing the EGFP locus (pMJ922, Addgene \#78312 [28]), 1 l Ribolock R1 RNAse inhibitor (Thermo Fisher, Landsmeer, the Netherlands) and filled to $30 \mu \mathrm{l}$ with nuclease-free water (Thermo Scientific, Landsmeer, the Netherlands). The reaction was completed in 2 hours at $37^{\circ} \mathrm{C}$. The samples were treated with $1 \mu$ l proteinase K (Thermo Fisher, Landsmeer, the Netherlands) 
and filled to $30 \mu \mathrm{l}$ with nuclease-free water (Thermo Scientific, Landsmeer, the Netherlands) and were separated using agarose gel (1\%) electrophoresis and visualized with 5 $\mu \mathrm{L}$ Midori Green (Nippon Genetics, Düren, Germany) staining per $100 \mathrm{~mL}$ of agarose. SpCas9 activity was calculated by gel densitometry, by determining the area under the curve in ImageJ, and calculating the relative cleaved fraction. This was repeated over the course of one year to determine the protein stability in storage.

\section{Lipid Nanoparticle Formulation}

To formulate LNP for gene knock-out (LNP-RNP), sgRNA and SpCas9 were mixed at a 1:1 molar ratio in different formulation buffers $(100 \mathrm{mM}$ citrate buffer $(\mathrm{pH} 4.0)$, Dulbecco's PBS (pH 7.4), 50 mM HEPES buffer (pH 7.4; LNP-RNP [HEPES]), or nuclease-free water at an RNP concentration of $0.4 \mu \mathrm{M}$. Complexation was performed for 15 minutes at room temperature. Concurrently, the lipids were mixed together in ethanol to achieve a total lipid to sgRNA ratio of 40:1 (w/w), resulting in a total lipid weight of $9.6 \mu \mathrm{g}$ [23]. The lipid components, represented in Fig. 2a, were C12-200, DOPE, cholesterol, PEG-DMG, and DOTAP (molar ratio 35:16:46.5:2.5:variable). Different molar ratios of DOTAP were tested to find the optimal amount for complexation with RNP. The RNP and lipids were mixed by pipetting at a volume ratio of 3:1 (18 $\mu \mathrm{l}$ RNP to $6 \mu$ lipids), and incubating for 15 minutes at room temperature. Subsequently, the formulation was diluted 4 times with PBS to a final RNP molar concentration of $76.9 \mathrm{nM}$ in $100 \mu \mathrm{l}$. Formulation steps with exact volumes are shown in table S.4.1.

LNP carrying RNP and HDR template (LNP-RNP-HDR) were formulated in the same manner in HEPES buffer or nuclease free water (LNP-RNP-HDR [HEPES] and LNPRNP-HDR $\left[\mathrm{H}_{2} \mathrm{O}\right]$, respectively), except that the HDR template was added at varying molar ratios of RNP:HDR template $(1: 2 ; 1: 3.8 ; 1: 5 ; 1: 10 ; 1: 20)$ to the RNP complex, prior to complexation with the lipids.

\section{Physical characterization of lipid nanoparticles}

LNP were diluted 1.3 times further in 1x PBS for characterization of size and polydispersity index (PDI) using a Zetasizer Nano S (Malvern ALV CGS-3, the United Kingdom). The $\zeta$-potential was determined with Zetasizer Nano Z (Malvern ALV CGS-3, United Kingdom) after 9x dilution in $10 \mathrm{mM}$ HEPES buffer at $\mathrm{pH} 7.4$. Each sample was measured in triplicate to determine size and $\zeta$-potential two days after formulation.

\section{Quantification of RNP complexed with LNP}

Complexation efficiencies were determined in LNP prepared in the different formulation conditions. RNP at $1.25 \mu \mathrm{M}$ and a final formulation volume of $0.47 \mathrm{~mL}$ in PBS were used. For determination of SpCas9 complexation, the LNP formulation was additionally dialyzed against 1x HBS with Float-A-Lyzer MWCO 300kDa dialysis chambers (Avantor®, the Netherlands) to remove free SpCas9 from the formulation.

Reversed-phase HPLC (Waters Alliance e2695) was performed to determine the amount of SpCas9 that was complexed with LNP, using an Xbridge protein BEH C4 $300 \AA$ column (Waters \#186004505) with a linear acetonitrile gradient, from $5 \%$ to $100 \%$ in 5 minutes and back again in 1 minute, with 10 minutes of total elution time. The mobile phase additionally contained $0.1 \%$ trifluoric acid. The column was heated at $30{ }^{\circ} \mathrm{C}$. Fluorescence detection was set at ex. $280 \mathrm{~nm}$, em. $350 \mathrm{~nm}$ (10pts/sec) and UV/Vis detection was set at 214 and $280 \mathrm{~nm}$ (2pts/sec). Samples were treated with $2 \%$ Triton X-100 for 5 minutes before injection. Samples were injected with an injection volume of $50 \mu \mathrm{l}$ and at a flow rate of $1 \mathrm{ml} / \mathrm{min}$. A calibration curve of empty LNP spiked with SpCas9, with a concentration range of $0-300 \mathrm{nM}$ and treated with $2 \%$ Triton X-100, was used to quantify the SpCas 9 concentration. 
The Quant-iTTM RiboGreen ${ }^{\circledR}$ RNA kit (Fisher Scientific, Landsmeer, the Netherlands) was used to determine the complexation efficiency of sgRNA. The protocol provided by the supplier was followed, except that sgRNA was used instead of the RNA standard to generate a calibration curve in RNAse-free TE buffer. A calibration curve with and without $2 \%$ Triton X-100 was made in duplicate. LNP samples and the calibration curve that were not treated with $2 \%$ Triton X-100 were treated with the same volume of $1 \times$ RNAse-free TE buffer. Fluorescence signal (ex. 485 nm, em. $520 \mathrm{~nm}$ ) was determined with Jasco FP8300 Spectrofluorometer with micro-well plate reader (JASCO Benelux BV., De Meern, the Netherlands).

\section{Stability of lipid nanoparticles in human plasma}

Stability of LNP was determined by asymmetric flow field flow fractionation (AF4) measurements using the AF2000 separation system (Postnova Analytics, Landsberg, Germany). The system is equipped with a degasser, isocratic pumps, auto samples, fractionation channels, and an in-line DLS detector (Zeta Nano ZS, Malvern Instruments). For separation, a FFF channel was used with a $350 \mu \mathrm{m}$ spacer and a regenerated cellulose membrane with a molecular weight cut-off of $10 \mathrm{kDa}$. PBS was used as mobile phase.

LNP-RNP [HEPES] and LNP-RNP-HDR [HEPES] or LNP-RNP-HDR [H20] were prepared as described above, with a total lipid concentration of $4.4 \mathrm{mM}$ and RNP concentration of 1.6 $\mu \mathrm{M}$. In addition, $3 \mu \mathrm{M}$ HDR template was added to the LNP-RNP-HDR formulation. LNP formulations were not diluted with PBS as described previously as high concentrations were needed for the AF4 studies. To verify potential destabilizing effects of blood components on the LNP, the nanoparticles were treated with $20 \%$ human plasma (\#HMPLCIT, BioIVT, West Sussex, United Kingdom) and incubated for $1 \mathrm{~h}$ at $37^{\circ} \mathrm{C}$. Subsequently, $20 \mu \mathrm{l}$ was injected at a flow rate of $0.2 \mathrm{ml} / \mathrm{min}$ and focused for 4 minutes with a cross-flow of $1.5 \mathrm{ml} / \mathrm{min}$ and a focus flow of $1.8 \mathrm{ml} / \mathrm{min}$. After 1 minute transition time, the cross flow was kept consistent at $1.5 \mathrm{ml} / \mathrm{min}$ for 5 minutes before it was decreased with a linear decay of 1 to $0.5 \mathrm{ml} / \mathrm{min}$ over a span of 25 minutes. Then, the cross flow was decreased with an exponential decay of 0.3 for 30 minutes until it reached $0 \mathrm{ml} / \mathrm{min}$, at which it was kept constant for 10 minutes. During the entire run, the detector flow rate was 0.5 $\mathrm{ml} / \mathrm{min}$.

\section{Cell Culture}

HEK293T stoplight cells [29] and HEK293T cells with stable EGFP expression were cultured in low-glucose DMEM medium supplemented with $10 \%$ fetal bovine serum (FBS), at $37{ }^{\circ} \mathrm{C}$ and $5 \% \mathrm{CO} 2$. For subculturing between experiments, $1 \mathrm{mg} / \mathrm{ml}$ Gibco® Geneticin ${ }^{\circledR}$ Selective Antibiotic (G418 sulfate, Fischer Scientific, Landsmeer, the Netherlands) was supplemented. Cell culture plastics were acquired from Greiner Bio-One (Alphen aan de Rijn, the Netherlands).

\section{Gene editing efficacy assays}

Stoplight gene editing assay: HEK293T stoplight cells were plated at a density of $3^{*} 10^{5}$ cells $/ \mathrm{cm}^{2}$ on a 96 -well black plate (Greiner CellStar \#655090). The following day, the cells were treated with $10 \mu \mathrm{l}$ of LNP-RNP supplemented with 1X Antibiotic Antimycotic solution (Sigma Aldrich, Zwijndrecht, the Netherlands). Cells were washed after 24 hours with $100 \mu \mathrm{l}$ of low-glucose DMEM medium supplemented with 10\% FBS and 1X Antibiotic Antimycotic solution. The cells were incubated for another 24 hours at $37{ }^{\circ} \mathrm{C}$ and $5 \%$ CO2. Following this, the cells were treated with $2 \mu \mathrm{g} / \mathrm{ml}$ Hoechst 33342 in complete cell culture medium for $15 \mathrm{~min}$ and imaged using the Yokogawa CV7000 Confocal Microscope (Yokogawa Corporation, Tokyo, Japan). Fluorescence image analysis was performed with the Columbus Software (Perkin Elmer, version 2.7.1), of which the analysis workflow is shown in Fig. S.6.6. Gene editing efficiency was defined as the number of cells expressing EGFP divided by the number of cells expressing mCherry, as described previously [29]. LNP formulations were compared to a positive control, consisting of RNP delivered using 
ProDeliverIN CRISPR (Oz Biosciences, San Diego, USA) as specified by the manufacturer, except that a $3.3 \mu \mathrm{l}: 1 \mu \mathrm{g}$ ratio of reagent to protein was used.

EGFP - BFP mutation assay: Mutation of the EGFP signal to BFP as measure of gene correction was based on the work of Glaser et al [30]. Briefly, HEK293T-EGFP cells were seeded at a density of $3^{*} 10^{5}$ cells $/ \mathrm{cm}^{2}$ in an appropriate cell culture plate. The following day, medium was supplemented with $1 \%$ antibiotic/antimycotic solution and LNP formulations were added to each well, containing a varied concentration of RNP, HDR template and lipid concentrations. As a positive control, ProDeliverIN CRISPR was used to deliver the RNP and the HDR template in a molar ratio of 15:15:28.5 nM. Cells were washed after 24 hours with fresh medium, and incubated for two days. Subsequently, they were passaged and expanded for two additional days, leading to a total of five days incubation after transfection. Cells were subsequently harvested, washed twice with PBS, fixed in $1 \%$ paraformaldehyde, and transferred to a BD Falcon U-bottom 96 well plate (Becton Dickinson, Franklin Lakes, USA).

Cell fluorescence was determined by flow cytometry using the BD FACS CANTO II (Becton Dickinson, Franklin Lakes, USA). BFP was measured using the Pacific Blue channel of the flow cytometer, while EGFP fluorescence was determined in the FITC channel. Data was analyzed with the Flowlogic software (Inivai Technologies, Mentone, Australia, version 7.3). Gene knock-out was defined as a loss of the green fluorescent signal, whereas gene correction was defined as a gain of the blue fluorescent signal. The gene editing efficiency was determined by the population negative for EGFP and BFP, indicating gene knock-out, as well as the population positive for blue fluorescence, indicating HDR correction using the specified template. A plasmid encoding this BFP plasmid is given in S.2.1, and was acquired from Twist Bioscience (San Francisco, USA). The gating strategy and model validation are presented in S.2.2.

\section{T7 Endonuclease Assay}

To validate the functional gene-editing readouts, a T7 endonuclease I (T7E1) assay was performed. Genomic DNA was extracted from HEK293T stoplight cells and HEK293T-EGFP cells 2 or 5 days after the transfection with LNP-RNP and LNP-RNPHDR, respectively, using the PureLink Genomic DNA Mini Kit (Thermo Fisher, Landsmeer, the Netherlands) following the manufacturer's instructions. PCR amplification was performed using primers designed specifically for the target locus (Supplementary information S.1.2) using Q5® Hot Start High-Fidelity 2X Master Mix (New England Biolabs, Ipswich, USA). Afterwards, PCR products were purified using QIAquick PCR Purification kit. PCR products were denatured at $95^{\circ} \mathrm{C}$ for $10 \mathrm{~min}$ in presence of NEBuffer 2 (New England Biolabs, Ipswich, USA) and annealed at $-2{ }^{\circ} \mathrm{C}$ per second temperature ramp to $85^{\circ} \mathrm{C}$, then at $-0.1^{\circ} \mathrm{C}$ per second temperature ramp to $25^{\circ} \mathrm{C}$. Following this, hetero-duplexed sequences were incubated with 5U T7E1 enzyme (New England Biolabs, Ipswich, USA) at $37^{\circ} \mathrm{C}$ for $18 \mathrm{~min}$ to achieve digestion of mismatched DNA.

\section{Results}

\section{SpCas9 production, characterization and stability in storage}

SpCas9 was recombinantly produced by transforming the LPS-free ClearColi ${ }^{\mathrm{TM}}$ BL21 strain with plasmid pET15_SpCas9_NLS_His (Addgene \#62731). The elution chromatogram of SpCas9, given in S.3.2, shows that the principal protein component elutes at 250 $\mathrm{mM}$ imidazole. To study the long-term stability of in-house produced SpCas9, purified SpCas9 from a representative batch was snap frozen in liquid nitrogen and stored in aliquots at $-80{ }^{\circ} \mathrm{C}$ until needed for analysis of protein size, activity, and for use in LNP formulations. As shown in Fig. 1a, the SpCas9 protein appeared as a clear band on SDSPAGE at the expected molecular weight of $160 \mathrm{kDa}$. The relative peak area of the principal SpCas9 band, calculated by SDS-PAGE densitometry, did not deteriorate over time, as 
shown in Fig. 1b (gel excerpts underlying this graph are given in S.3.3). SpCas9 furthermore proved to be active at introducing a targeted double strand break in plasmid DNA only when complexed with the cognate sgRNA as seen in agarose gel electrophoresis in Fig. 1c. This activity was retained over time, as an activity digest after 12 months of storage showed similarly high SpCas9 activity. The activity did not differ significantly from the positive commercial control for each assay performed over time (Fig. S.3.1). These results show that the recombinant SpCas9, produced and stored with these methods and conditions, was active and stable at least for one year. This recombinant SpCas9 was used in subsequent formulation and gene editing studies.
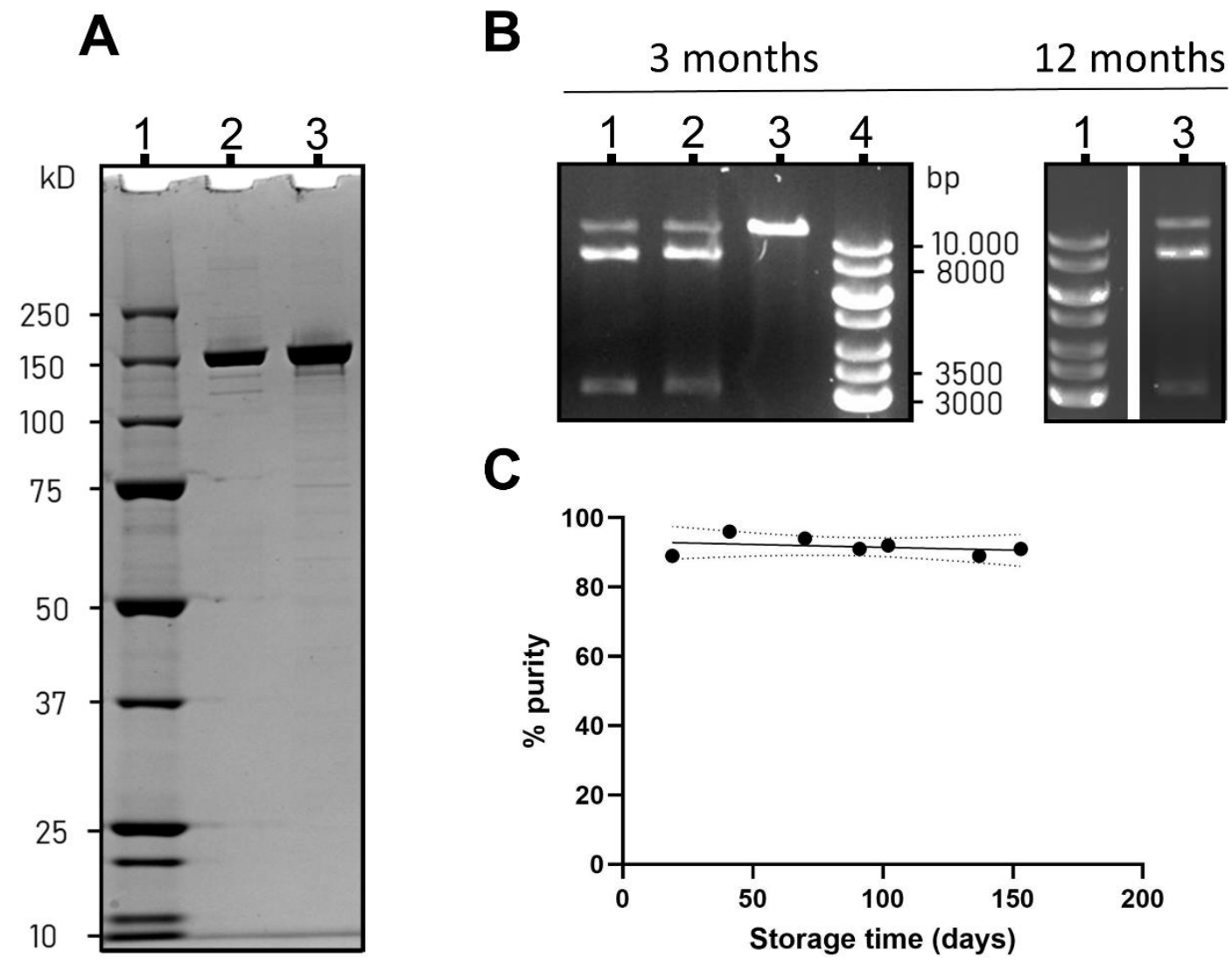

Figure 1: SpCas9 characterization after purification and extended storage. A: SDS-PAGE gel of the purified recombinant SpCas9. 1: PageRuler Plus prestained protein ladder. 2: Positive control SpCas9 acquired from Sigma Aldrich. 3: In-house produced SpCas9. B: Relative density of the $160 \mathrm{kDa}$ protein band on the SDSPAGE gels over time, defined as percentage purity. C: Activity of the SpCas9 protein (lane 1 left gel, lane 3 right gel) compared to a commercial sample (lane 2, left gel) and a negative control (lane 3, left gel). Generuler $1 \mathrm{kB}$ ladder (lane 4 left gel, lane 1 right gel) was used for determining the size of the DNA fragments. The activity is shown for SpCas 9 after 3 months and 1 year in storage. 


\section{Characterization and efficacy of LNP formulations for gene knock-out (LNP-RNP)}

Since $\mathrm{pH}$ and ionic strength may influence Cas9 RNP activity as well as RNP complexation during LNP preparation, different LNP formulations for gene knock-out were prepared by varying buffer composition during complexation of RNP with lipids, as well as the total amount of DOTAP in the final LNP-RNP formulations (Fig. 2). LNP consistently showed a particle size between $100 \mathrm{~nm}$ and $200 \mathrm{~nm}$ and a PDI below 0.2 , as well as a $\zeta$-potential between -5 and -20 $\mathrm{mV}$ (Fig. 2b,c). Interestingly, the LNP-RNP formulation prepared with nuclease-free water in the complexation phase, and containing 5 mole\% DOTAP seems to result in a high average particle size and polydispersity index ( $1000 \mathrm{~nm}$, PDI 0.8$)$, suggesting this formulation is colloidally unstable, leading to LNP aggregation. A larger polydispersity index was additionally determined for LNP-RNP formulated in nuclease-free water with 2 mole\% DOTAP. Quantification of the amount of SpCas9 protein and sgRNA associated with the LNP was done with HPLC and Quant-iTTM RiboGreen ${ }^{\circledR}$ RNA assay, resulting in complexation efficiencies of $63.7 \%$ and 68.6 $\%$ (formulation: 5 mole\% DOTAP, $50 \mathrm{mM}$ HEPES buffer for RNP complexation), respectively (Fig. S.5.1. and S.5.2.). As RNP is a 1:1 complex of gRNA to SpCas9 protein a similar complexation efficiency to lipid nanoparticles is expected, as validated by studying both SpCas9 and gRNA. Thus, complexation of SpCas9 was used in a further study to compare the effect of formulation buffer on RNP complexation in LNP and interestingly no differences could be detected (Fig. S.5.3).

To determine LNP-RNP stability under near-physiological conditions, AF4 was applied to detect intact LNP and measure its average size distribution when incubated in $5 x$ diluted human plasma. The formulations tested during AF4 studies were LNP-RNP [HEPES], containing 0 and 5 mole\% DOTAP. Depicted in Fig. 2 are fractograms detected by in-line DLS detectors (Fig. 2d,e). LNP show a retention time around 40 minutes. The peaks on the DLS fractograms of nanoparticles incubated with plasma over the range of the retention times between 10 and 20 minutes are likely to be plasma proteins, suggested by an overlay of the chromatogram of $20 \%$ human plasma (S.7.1). LNP-RNP particles show a significantly higher derived count rate after incubation with plasma (Fig. 2d). These results indicate that these LNP do interact with the plasma components, suggesting formation of a protein corona on the surface of the LNP [31]. Based on these findings on particle size, RNP-lipid complexation efficiency and stability, the particles were deemed suitably stable and monodisperse to be tested on reporter cell lines for their gene editing efficiencies. 

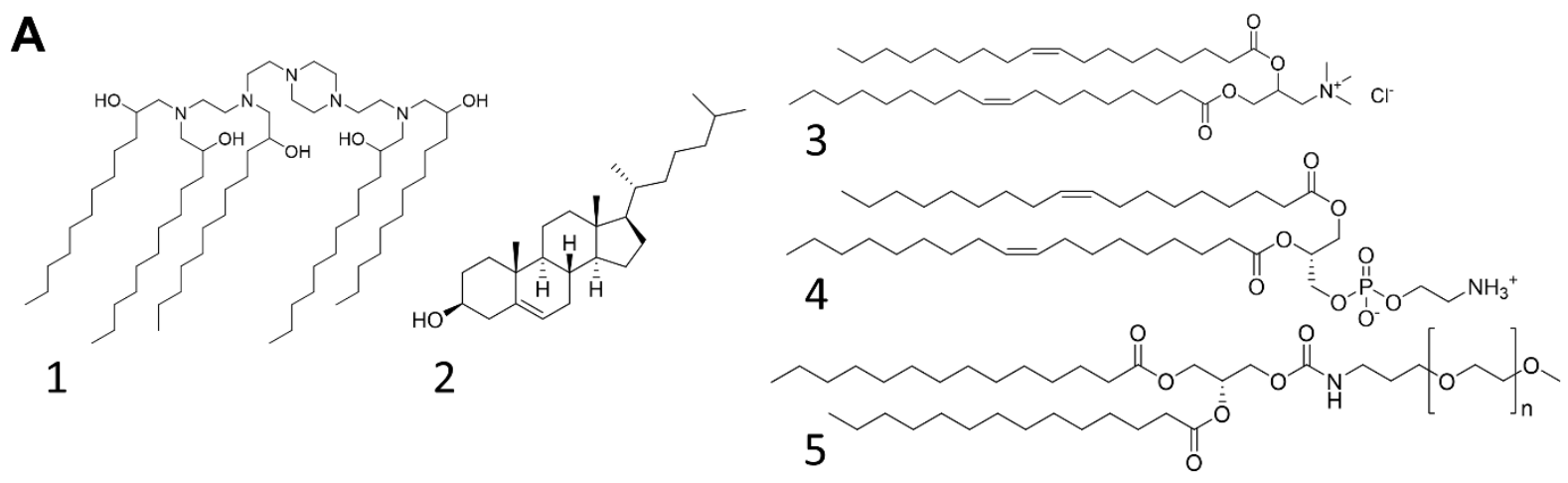

B
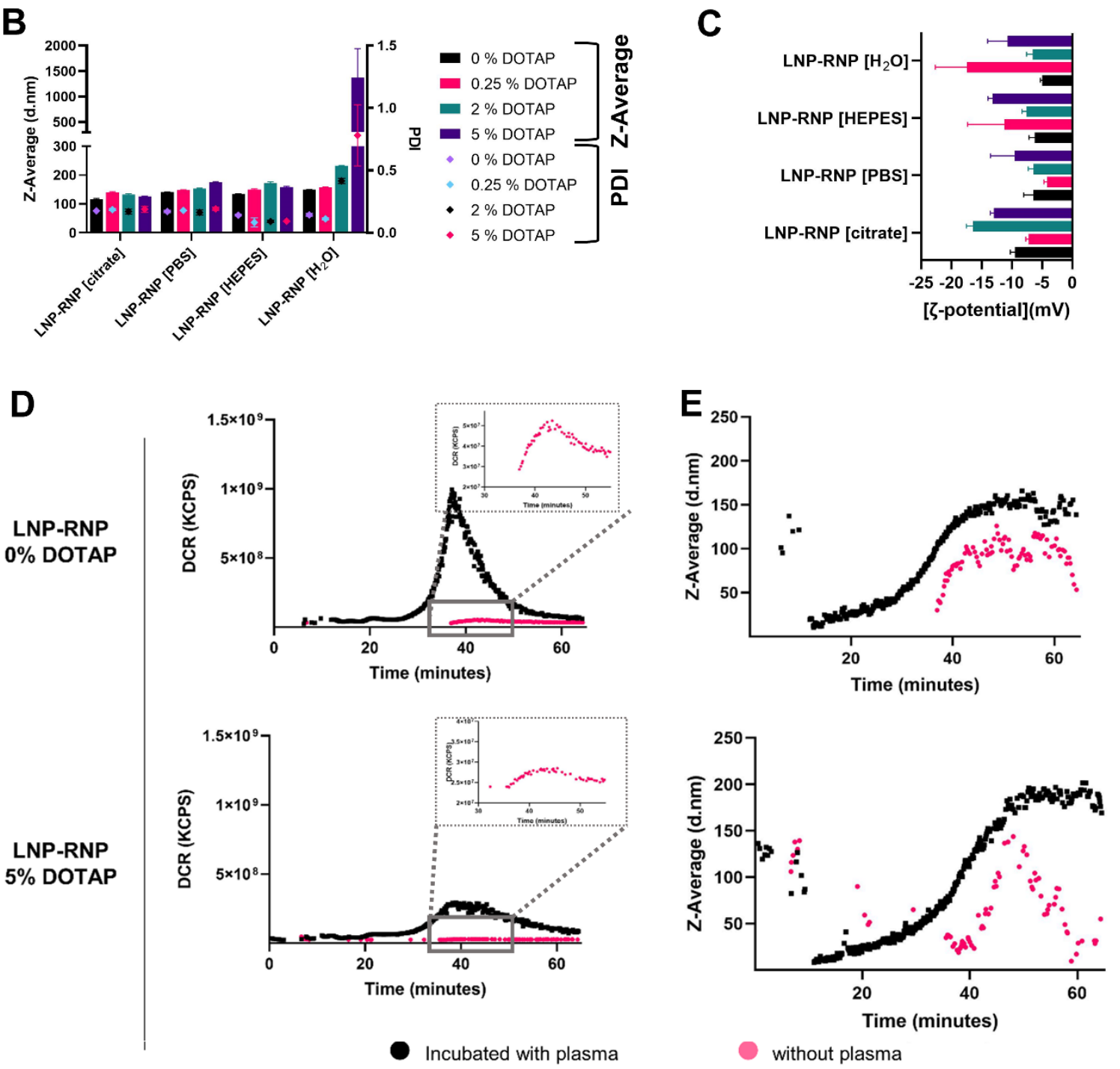

Figure 2: LNP characterization and plasma stability. A: Chemical structures of the LNP components in the formulations. 1 : C12-200, 2: cholesterol, 3: DOTAP, 4: DOPE, 5: PEG-DMG. B and C: LNP-RNP characteristics screened for varying DOTAP concentrations and complexation buffers. B: Average particle size and PDI in PBS as determined by DLS (measured in triplicate). C: $\zeta$-potential of these formulations in $10 \mathrm{mM}$ HEPES buffer $\mathrm{pH} 7.4$ (measured in triplicate). Two of these formulations were further characterized on stability in plasma (AF4). D,E: AF4 fractograms recorded by DLS detector showing the derived count rate (D) and particle size (E) for LNP-RNP formulated in HEPES buffer with 0 and 5 mole\% DOTAP. Inserts show a zoomed-in version of the samples measured without plasma. Detector flow was set to $0.5 \mathrm{ml} / \mathrm{min}$. 


\section{Determination of gene knock out efficiency of different LNP-RNP formulations}

LNP were applied to the HEK293T stoplight cell line to determine functional delivery of RNP. These cells constitutively express mCherry and, upon introduction of a +1 or +2 frameshift targeted by CRISPR-Cas9 downstream of the mCherry coding sequence, co-expression of EGFP is induced [29]. The influence of buffer composition during RNP formation was first assessed, as acidic buffers were shown to be detrimental in past reports $[15,23]$. Based on EGFP expression percentages, RNP formed in $50 \mathrm{mM}$ HEPES buffer ( $\mathrm{pH}$ 7.4) or nuclease-free water resulted in much higher gene editing in comparison to citrate or PBS buffer (Fig. 3a,c). This was confirmed at the genetic level using the T7E1 assay (Fig. 3e, S.6.4). An acidic environment clearly has a negative effect on RNP and LNP formation in accordance with the literature [23]. Contrary to these findings, however, limited editing activity was observed in PBS, which is a physiological buffer system. An in vitro activity assay was performed to investigate these effects further. These assays showed that complexation in PBS and citrate leads to irreversible inactivation of the RNP at a DNA-cleavage level (Fig. 3b, Fig S.3.4.b). In contrast, RNP mixed at different $\mathrm{NaCl}$ concentrations (up to $1 \mathrm{M}$ ) did not lose activity (Fig. S.3.4.b). Taken together these findings indicate that $\mathrm{pH}$ or ionic strength alone do not account for the loss of Cas9 activity in the formulations.

The gene knock- out efficiencies determined by flow cytometry were consistently lower than those determined by image analysis. The higher values obtained with image analysis can be explained by false positives due to difficulties in segmenting individual cells in highly confluent cell images. Nonetheless, flow cytometry confirmed that complexation of the RNP and LNP in HEPES buffer or nuclease-free water are the preferred complexation conditions. As LNPRNP formulations still have approximately $30-40 \%$ of free RNP that was not removed prior to transfection, LNP-RNP transfection efficiencies were compared before and after dialysis overnight against $1 \times$ HBS using a $300 \mathrm{kDa}$ MWCO dialysis membrane to remove free RNP. No difference in gene knock-out efficiency was observed (Fig. S.6.3b), indicating that gene editing was primarily caused by the RNP complexed to LNP. 
A

\section{Citrate buffer Heps buffer $\quad$ Nuclease-free water}

LNP-RNP with 0\% DOTAP
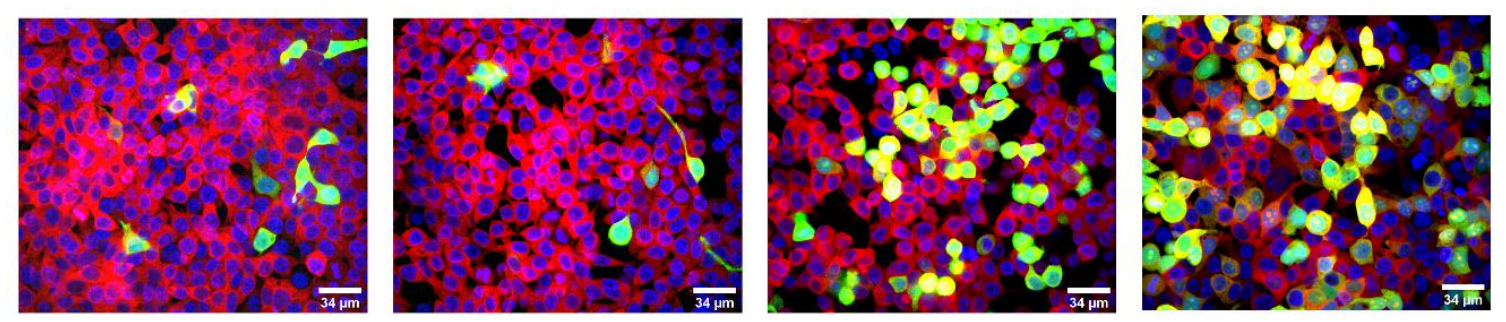

LNP-RNP with 5\% DOTAP
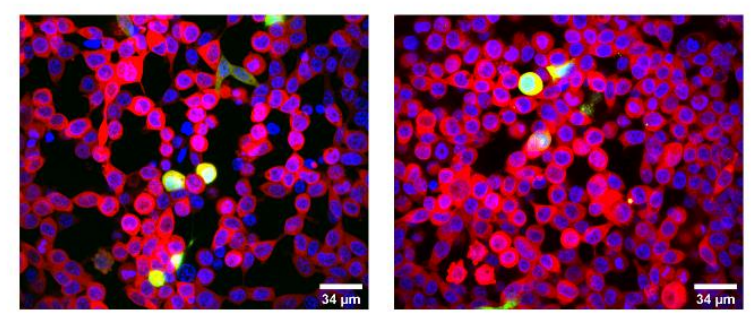

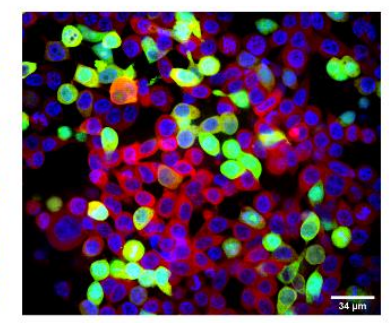

D
B

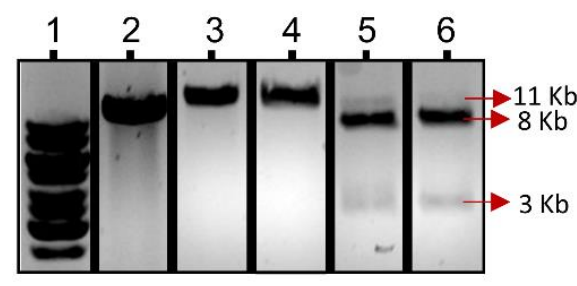

E
C

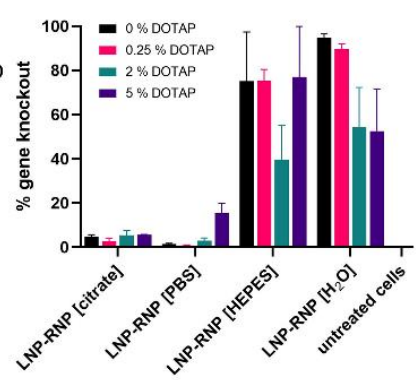

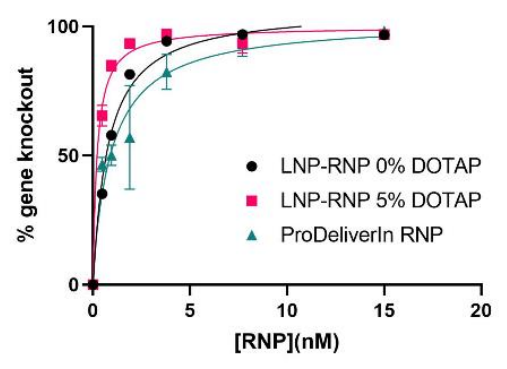

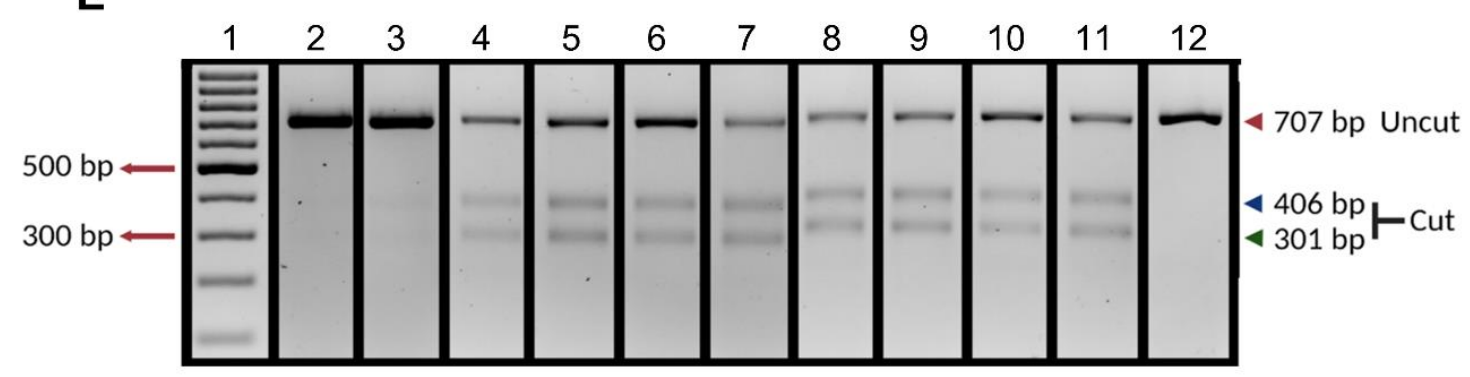

Figure 3: Determination of gene knock-out efficiency in HEK293T stoplight cells. A: Confocal Microscopy Images (60x) of HEK293T stoplight cells after treatment with different LNP formulations at a RNP concentration of $7.7 \mathrm{nM}$ (RNP were complexed in different conditions: $100 \mathrm{mM}$ citrate buffer, PBS buffer, $50 \mathrm{mM}$ Hepes buffer, nuclease-free water). Red - mCherry, green - EGFP (Cas9 gene editing), blue - Hoechst (nucleus). Scale bar $34 \mu \mathrm{m}$. Images were optimized on ImageJ in brightness and contrast for each channel, respectively. B: Cas9 activity in vitro using the same buffers as in A during RNP complexation. uncut (11 kB) and cut (8kB and $3 \mathrm{kB})$ DNA are highlighted by arrows. 1: Generuler 1 kB DNA ladder, 2: Untreated DNA. 3-6: RNP complexed in citrate (3), PBS (4), HEPES (5) or water (6). C: Gene knock-out efficiencies for different LNP formulations (with final RNP concentration $7.7 \mathrm{nM}$ ) determined by confocal image analysis using Columbus ${ }^{\circledR}$ software (tested in triplicate). D: Dose-dependent gene knock-out efficiencies of two selected LNP-RNP formulations (0 \% DOTAP and $5 \%$ DOTAP, 50 mM HEPES buffer) in comparison to the commercial transfection agent, ProDeliverIN (tested in duplicate). E: T7E1 digests performed on the same samples, and ordered as in panel C. 1: DNA ladder. 2: LNP-RNP containing 5 mole\% DOTAP, prepared in $100 \mathrm{mM}$ citrate buffer. 3: LNP-RNP containing 5 mole\% DOTAP, prepared in PBS. 4-7: LNP-RNP prepared in 50 mM HEPES buffer with 0, 0.25, 2 and 5 mole\% DOTAP, respectively. 8-11: LNP-RNP prepared in water with $0,0.25,2$ and 5 mole\% DOTAP, respectively. 12: negative control. The unedited gel is provided in S.6.4 as the order of the lanes was changed for clarity within this figure. 
A 3-way ANOVA was performed to statistically determine the effect of formulation condition, experimental repeat, and molar ratio of DOTAP on gene knock-out efficiency. Based on the statistical analysis the LNP-RNP formulation using nuclease-free water resulted in significantly higher gene editing outcomes compared to those prepared in HEPES buffer (Fig. S.6.5). This result depended on the molar ratio of DOTAP used during nanoparticle formulation as well (Fig. S.6.5), indicating that RNP and LNP complexation in HEPES buffer requires higher mole\% DOTAP than in water. The statistical analysis however does show batch variation from one experiment to another, especially between formulations with HEPES buffer.

Dose-dependent gene knock-out was studied with two formulations complexed in $50 \mathrm{mM}$ HEPES buffer pH 7.4 and with LNP containing 0 or 5 mole\% DOTAP (Fig. 3d). From these results, the concentration to reach $50 \%$ of the effect (EC50) was calculated as a measure of gene knock-out efficiency by fitting a dose-response curve ([Agonist] vs response) using GraphPad PRISM version $9.1\left(\mathrm{r}^{2}\right.$ for LNP-RNP 0\% DOTAP = 0.98; $\mathrm{r}^{2}$ for LNP-RNP 5\% DOTAP = 0.99; $\mathrm{r}^{2}$ for ProDeliverIN RNP $=0.93)$. LNP formulated with $0 \%$ DOTAP have a higher EC50 value $(0.8 \mathrm{nM})$ than the formulation with 5\% DOTAP $(0.2 \mathrm{nM})$. In comparison, the fit led to an EC50 value of $1 \mathrm{nM}$ for the ProDeliverIN positive control. In conclusion, therefore, LNP-RNP with 5 mole\% DOTAP formulated in HEPES buffer seems to be the best performing nanoparticle for gene knock-out. Incubation of HEK293T stoplight cells with LNP-RNP did not result in any cytotoxicity at an RNP concentration around $7.7 \mathrm{nM}$ (Fig. S.6.1.a). Incubation of cells with $15 \mathrm{nM}$ of LNP-RNP did result in a lower absolute number of cells (Fig. S.6.1.b).

\section{Characterization of LNP formulations for gene correction (LNP-RNP-HDR)}

LNP formulations additionally containing a single stranded DNA template for HDR-mediated gene-correction were optimized using a similar rationale as the LNP-RNP formulations. Water and HEPES buffer at $\mathrm{pH} 7.4$ were selected as primary formulation conditions following the LNP-RNP screening. Further variables were molar ratio of RNP to HDR template, and mole $\%$ of DOTAP in the LNP composition. To determine whether ssDNA HDR template has an effect on size and $\zeta$-potential, these values were determined for formulations prepared in HEPES buffer, as differences amongst formulation conditions were not expected as shown in figure 2. Their characteristics were similar to those found for LNP-RNP (Fig. 4a,b), except for the formulation with a 1:1 ratio RNP:HDR, which resulted in a higher polydispersity index. The $\zeta$-potential of these particles was interestingly similar to that of the LNP-RNP particles, even though more anionic charges are added to the formulation (up to 10-fold molar excess of template DNA compared to RNP).

The plasma interaction of these particles was additionally tested using AF4. The results of the LNP-RNP-HDR particle formulated in nuclease-free water remarkably do not show a shift in retention time, as opposed to LNP-RNP (Fig 4c, d). Interestingly, the increased count rate after plasma incubation is less pronounced in particles entrapping HDR template. Moreover, the particles additionally entrapping an HDR template do not change in size (Fig. 4d) [32,33]. 
A

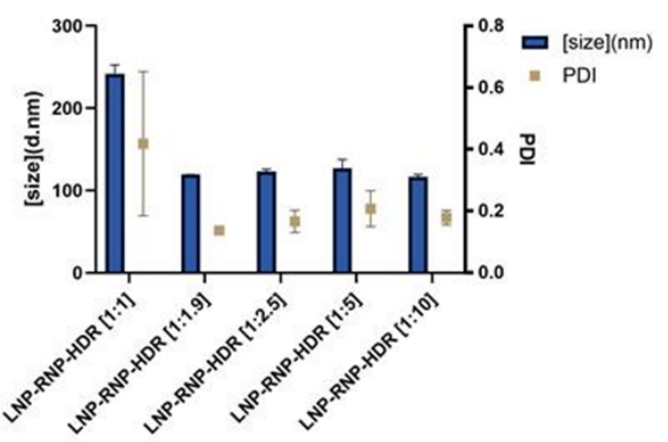

C

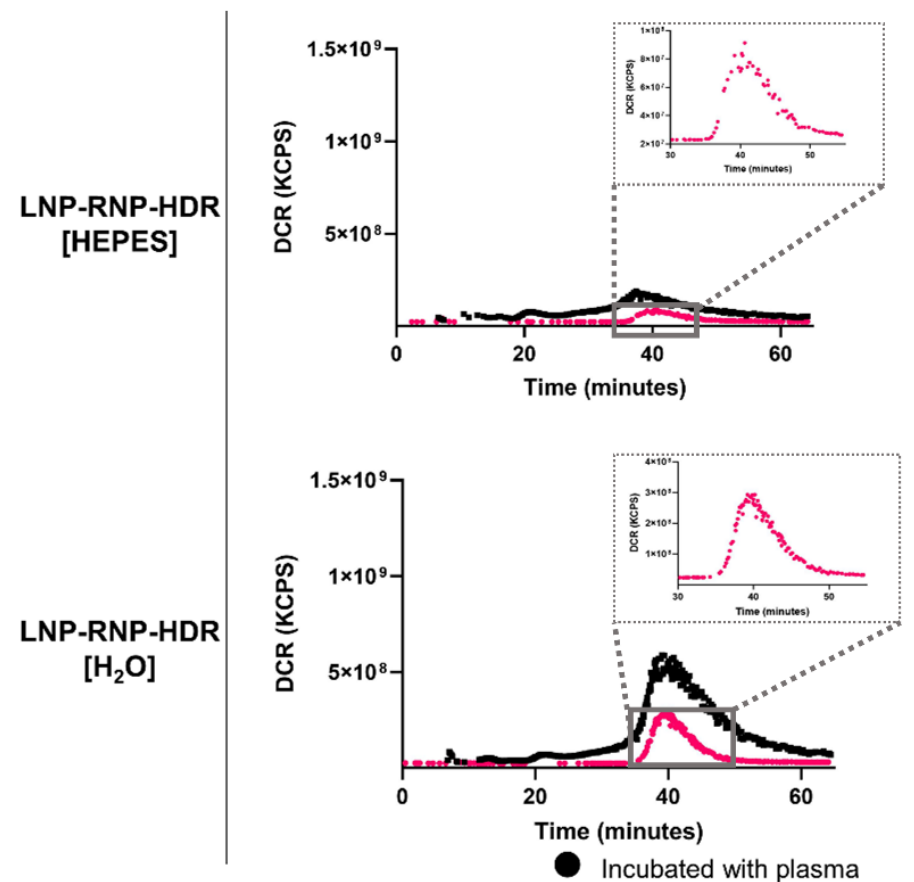

B

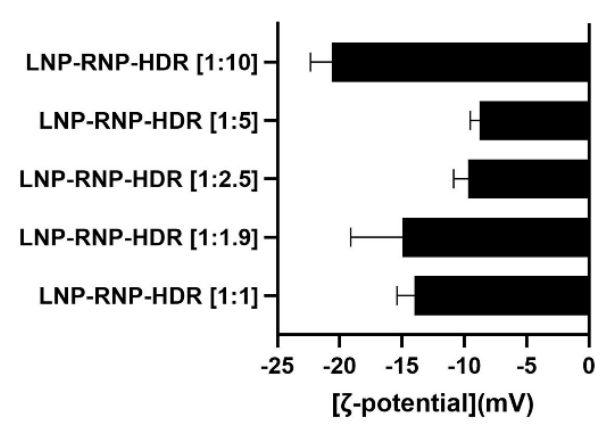

D
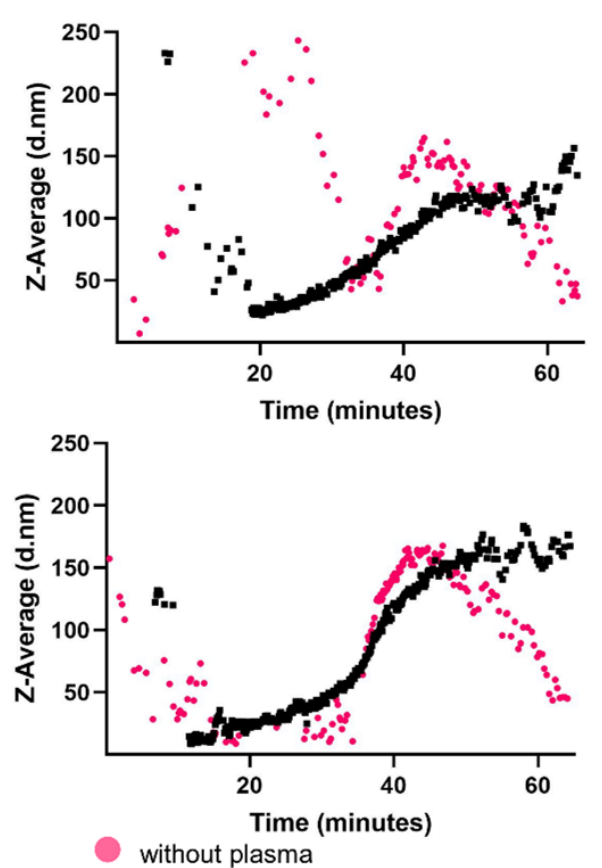

without plasma

Figure 4: Characterization of LNP-RNP-HDR formulations. A and B: Representative LNP-RNP-HDR characteristics screened for varying HDR template concentrations (in molar ratios compared to RNP) at a fixed complexation buffer (50 mM HEPES) and at a fixed lipid composition ( 0.25 mole\% DOTAP). A: Average particle size and PDI as determined by DLS (measure in triplicate). B: $\zeta$ potential of these formulations in $10 \mathrm{mM}$ HEPES buffer $\mathrm{pH} 7.4$ (measured in triplicate). C: AF4 fractograms recorded by DLS detector showing the derived count rate (DCR) of LNP-RNP-HDR formulations at a fixed HDR template concentration (1:1.9 molar ratio) and DOTAP concentration $(0.25$ mole $\%)$ in varying complexation buffers, with and without plasma incubation. D: AF4 fractograms recorded by DLS detector of particle size for LNP-RNP-HDR (same formulations as in C). Detector flow was set to $0.5 \mathrm{ml} / \mathrm{min}$. 


\section{Determination of gene correction efficiency of different LNP-RNP-HDR formulations}

LNP-RNP-HDR were tested for their gene-editing efficacy on HEK293t cells with constitutive EGFP expression. Loss of the EGFP indicates gene knock-out, while the rise of a blue signal indicates gene correction (S.2.2). Several concentrations of HDR template were screened (Fig. $5 a, b$ ), as well as DOTAP percentages. Leaving out DOTAP from the formulation led to a significant reduction in gene-editing efficiency (S.8.1). The formulation yielding the highest gene correction efficacy were the LNP prepared in water, which contained 0.25 mole\% DOTAP at a 1:2 molar ratio of RNP to HDR template. This formulation yielded a gene correction efficacy of $11.4 \%$ of the total cell population, as well as a gene knock-out efficacy of $59.6 \%$ of the cells at a final RNP concentration of $7.7 \mathrm{nM}$. For the LNP formed in HEPES buffer, the overall gene correction efficacies were lower. The percentage of HDR events within the total gene editing outcomes is given in Fig. 5b. This percentage is consistently higher for particles complexed in water compared to HEPES buffer, which indicates that the particles formulated in water were overall more suited for HDR. Another trend is that addition of higher relative concentrations of HDR-template is associated with lower gene-editing.

A dose-escalation study was performed for LNP-RNP-HDR formulations prepared in water or HEPES buffer with 0.25 mole\% DOTAP and a 1:2 ratio of RNP:HDR template, which performed well in the screening. The dose-dependent toxicity of these formulations after one day was assessed by the MTS assay (Fig. 5c). Cell viability decreased slightly over the concentration range but stayed above $90 \%$ along the whole concentration range for both formulations. The dose-dependent efficacy was determined by fitting a dose-response curve ([Agonist] vs response) using Graphpad PRISM version 9.1 for both gene correction $\left(\mathrm{r}^{2}\right.$ for LNP-RNP-HDR $[\mathrm{H} 2 \mathrm{O}]=0.96 ; \mathrm{r}^{2}$ for LNP-RNP-HDR $[$ HEPES] $=0.79$ ) and gene knock-out ( $\mathrm{r}^{2}$ for LNP-RNP-HDR [H2O]= $0.97 ; \mathrm{r}^{2}$ for LNP-RNP-HDR [HEPES] $=0.86$ ). These curves showed that formulations prepared in water exhibited a lower EC50 $(7 \mathrm{nM})$ for gene correction compared to the particles prepared in HEPES buffer $(47 \mathrm{nM})$. For gene knock-out, the EC50 was lower for all conditions, but the same trend was observed where the water particles show a lower EC50 $(1 \mathrm{nM})$ than HEPES particles $(10 \mathrm{nM})$ (Fig. $5 \mathrm{~d})$. Gene editing was additionally confirmed by the T7E1 assay (S.8.2.), indicating that cells in this population contained insertions or deletions in their genome. These data combined showed that LNP-RNP-HDR formulated in water reached a gene correction efficacy of $19.2 \%$ at a concentration of $15 \mathrm{nM}$ RNP with good cytocompatibility (95\% cell viability). 
A
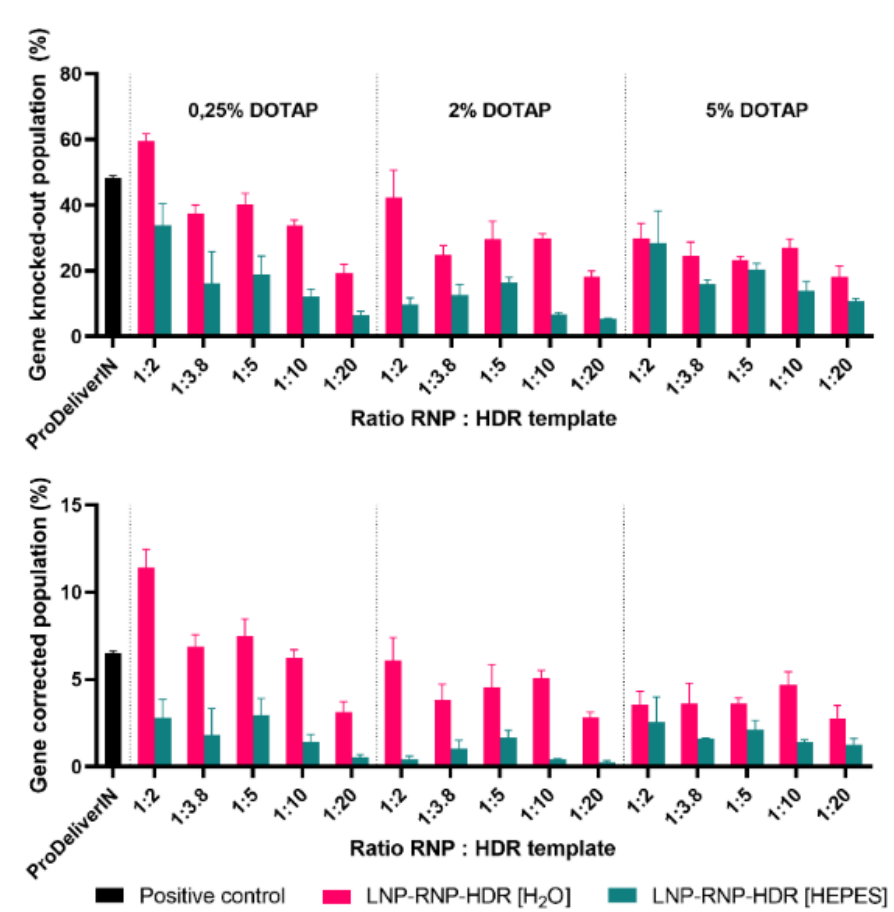

C

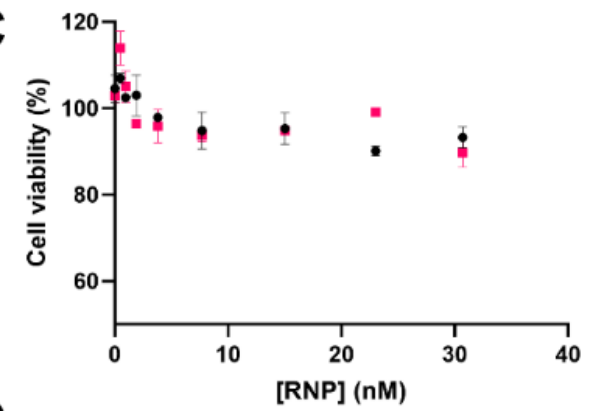

D

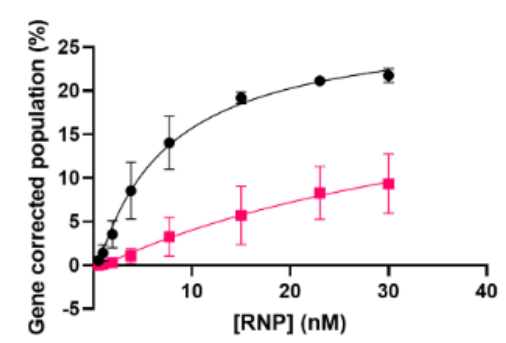

B

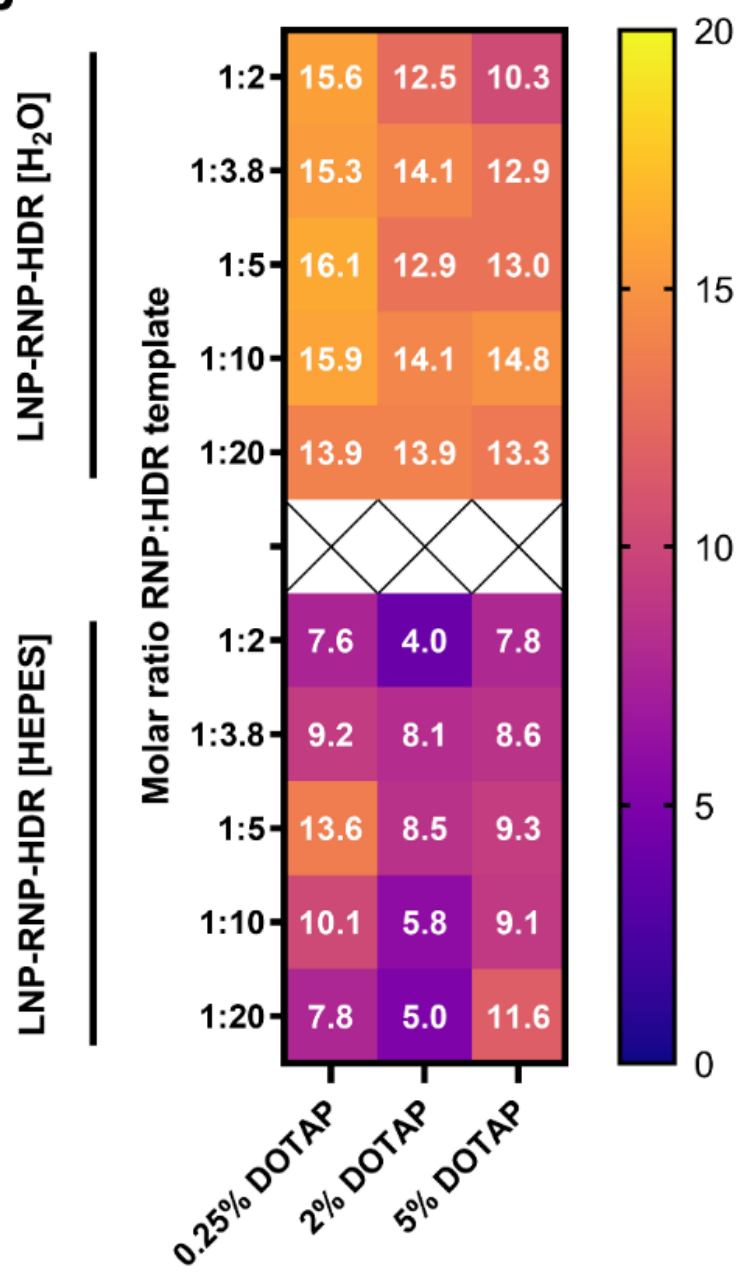

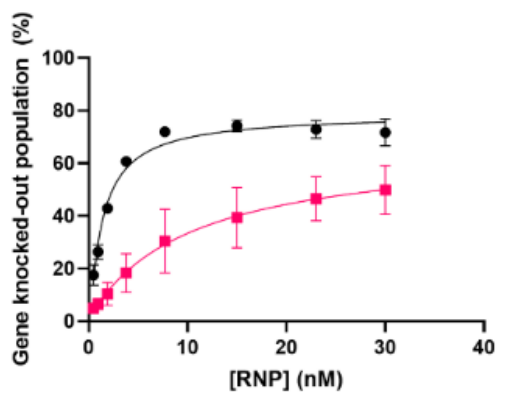

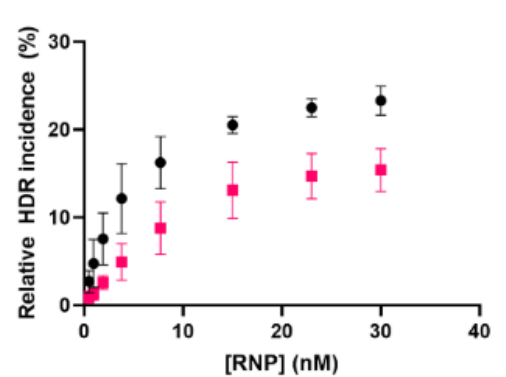

Figure 5: A: Formulation optimization to achieve gene-correction using LNP at an RNP concentration of $7.7 \mathrm{nM}$, with varying molar ratios of RNP:HDR template and percentages of DOTAP in the lipid composition (tested in triplicate). Complexation of RNP and lipids was performed in water or HEPES prior to transfection. The concentration of DOTAP and template DNA was varied. B: Heatmap representation of the relative gene correction ratio (percentage incidence compared to the sum of outcomes) within the gene-edited populations of Fig. 5a. C: MTS cell viability of a dose range of the best performing formulations formed in HEPES buffer (pink) or water (black), containing $0.25 \%$ DOTAP and a 1:2 molar ratio of HDR template to RNP (tested in duplicate). D: Dose escalation study performed with same formulations in Fig. 5c (pooled data from 2 batches; pink: HEPES buffer; black: Water), represented for the gene correction, gene knock-out and relative incidence of HDR as percentage within the gene-edited population (tested in duplicate). 


\section{Discussion}

We based our formulations on previous literature regarding LNP for mRNA delivery as starting point $[13,34,35]$. These use ionizable lipids to simultaneously reduce toxicity, facilitate nucleic acid entrapment and endosomal escape in target cells. Our findings support previous reports, showing that for complexation of RNP, the buffer during RNP and lipid nanoparticle complexation and the inclusion of cationic lipid DOTAP are necessary for stable particles [23]. We, however, further optimized these conditions for additional complexation of HDR template DNA. Buffer composition during RNP complexation played a major role on its downstream effect on cells. This seems not to be due to Cas9 encapsulation (Fig. S.5.3), but rather the Cas9 bioactivity as shown on in vitro gel digests (Fig. $3 b$ ). Whereas the RNP formed in water or HEPES was active, the RNP formed in citrate was not. Citrate in particular was tested, as it is used for lipid ionization in reported LNP formulations in the past. To our surprise, in vitro activity of SpCas9 RNP in PBS was severely reduced as well. This suggests that the inhibitory effect is not due to $\mathrm{pH}$ or ionic strength during complexation, but rather a specific buffer ion interaction. We showed that HEPES, for example, did yield active RNP in our particles. Further investigation of this effect may reveal buffer incompatibilities of Cas9 RNP.

Another interesting finding is the negative $\zeta$-potential. An explanation for the observed negative $\zeta$-potential could be adsorption of excess RNP to the surface of the LNP [36]. Interestingly, the addition of HDR template does not seem to change particle size or shift the $\zeta$-potential further toward negative, indicating that these are not surface-bound (Fig. 4a,b).

The efficacy of our optimized particles is in line with existing literature. Efficiency in gene editing seems to saturate around a concentration 5-10 nM RNP, thus higher concentrations of particles would not be required (Fig. 3d). In comparison, Suzuki et al. have shown editing saturation at $1 \mathrm{nM}$ with their lipid nanoparticles, however formulation conditions are not comparable to the conditions reported in this study [15]. Interesting to note also is that the incidence of NHEJ-based gene knock-out is more efficient in these formulations than HDR-based gene correction. This ratio, indicated in Fig. 5d, needs significant improvement before HDR can be considered for clinical application.

Finally, the AF4 studies show interesting insights on particle stability and potential protein corona formation in the presence of human plasma. More elaborate studies would need to be performed to verify the nature and content of such a protein corona and possibly specify which plasma proteins accumulate on the surface of the particles. Such investigations would be relevant, as a protein corona could mediate specific in vivo localization of the LNP, for example the adsorption of apolipoprotein E to the surface of LNP results in hepatocyte-specific uptake [33]. Incubation with plasma shifts the retention time of LNP formulated without a ssDNA HDR template to a slightly earlier retention time (Fig. 2d, $4 c$, and S.7.1). This indicates a change in the particle morphology due to interaction with plasma, worth investigating further, and that the HDR template may have a positive influence on particle stability [32,33]. Previous studies have shown that in fact additional anionic charges favour RNP stability in formulations, resulting in better gene editing efficiencies on cells after delivery via electroporation [37]. In any case, these results suggest that the particles are stable for in vivo applications and thus warrant further in vivo experimental studies. 


\section{Conclusions}

In this study, we set out to find optimized formulation conditions for LNP containing SpCas9 RNP, with and without HDR template. Our main findings are as follows:

i) Preparing RNP for formulation in nuclease free water or HEPES buffer yielded superior gene-editing results compared to PBS or citrate buffer, due to inadequate formation of an active RNP complex in the latter two buffers. There was no marked difference in encapsulation efficiency of Cas9 between these tested systems;

ii) incorporation of DOTAP in the LNP-RNP formulation was associated with a high gene-editing efficacy overall, while for LNP-RNP-HDR a lower concentration was optimal;

iii) high gene knock-out efficacies above $80 \%$ were achieved for LNP-RNP prepared in HEPES buffer, with 5 mole\% DOTAP, with a clear dose-dependent relationship;

iv) and as a highlighted result, 20\% gene correction efficacy was achieved with LNPRNP-HDR formulated in nuclease free water, 0.25 mole $\%$ of DOTAP and a 2:1 ratio of HDR template to RNP, with a clear dose dependent relationship as well, and high cell viability $(>90 \%)$.

Moreover, we demonstrate that these LNP formulations remain colloidally stable in the presence of human plasma, yet changes in scattering intensity and average size were detected, which might indicate formation of a protein corona on the particle's surface. Additionally, we provide a protocol for in-house production, purification and long-term storage of the SpCas 9 protein, which can be stored for at least a year at $-80{ }^{\circ} \mathrm{C}$ without loss of activity. These findings contribute to understand the necessity of optimal formulation conditions to create LNP for direct in vivo delivery of CRISPR-Cas9 components.

Supplementary Materials: Supplementary materials are available online at www.mdpi.com/xxx/s1.

Author Contributions: J.W and D.W contributed equally to the manuscript. J.W set up the methodology, performed investigation, data visualization and writing for the LNP formulation, characterization and gene knock-out studies. D.W set up the methodology, performed investigation, data visualization and writing for the Cas9 production and characterization and HDR-related experiments. V.S.J.T performed additional investigation and data visualization for the Cas9 stability study. M.O performed T7E1 and TIDE experiments. H.v.d.V set up the methodology for the Cas9 encapsulation efficiency study. Both B.L and E.M provided supervision and aided in the conceptualization and substantial writing of the manuscript.

Funding: This research was funded by the Netherlands Organisation for Scientific Research (NWO) Talent program VICI, grant number: 865.17.005.

Acknowledgments: The authors thank Lucas Czentner Colomo, Dr. Cornelis F. van Nostrum and Mies van Steenbergen for their help with the asymmetric flow field flow fractionation studies and data interpretation. In addition, the authors thank Willemijn de Voogt and Nanette Becht for their work on the SpCas9 production protocol The authors thank Erik R. Hebels for his support with the chemical structures made via ChemDraw. The support in statistical analysis by Dirk Walther is appreciated. Finally, we express our gratitude towards Olivier de Jong for providing us with and aiding us in the reporter cell lines.

Conflicts of Interest: The authors have no conflicts of interest to declare. 


\section{References}

1. Jinek, M.; Chylinski, K.; Fonfara, I.; Hauer, M.; Doudna, J.A.; Charpentier, E. A Programmable Dual-RNAGuided DNA Endonuclease in Adaptive Bacterial Immunity. Science (New York, N.Y.) 2012, 337, 816-821, doi:10.1126/science.1225829.

2. Gasiunas, G.; Barrangou, R.; Horvath, P.; Siksnys, V. Cas9-CrRNA Ribonucleoprotein Complex Mediates Specific DNA Cleavage for Adaptive Immunity in Bacteria. Proceedings of the National Academy of Sciences 2012, 109, E2579 LP-E2586, doi:10.1073/pnas.1208507109.

3. Doudna, J.A.; Charpentier, E. Genome Editing. The New Frontier of Genome Engineering with CRISPR-Cas9. Science (New York, N.Y.) 2014, 346, 1258096, doi:10.1126/science.1258096.

4. Salsman, J.; Masson, J.-Y.; Orthwein, A.; Dellaire, G. CRISPR/Cas9 Gene Editing: From Basic Mechanisms to Improved Strategies for Enhanced Genome Engineering In Vivo. Current Gene Therapy 2017, 17, 263-274, doi:10.2174/1566523217666171122094629.

5. Yang, H.; Ren, S.; Yu, S.; Pan, H.; Li, T.; Ge, S.; Zhang, J.; Xia, N. Methods Favoring Homology-Directed Repair Choice in Response to Crispr/Cas9 Induced-Double Strand Breaks. International Journal of Molecular Sciences 2020, 21, 1-20, doi:10.3390/ijms21186461.

6. Lieber, M.R. The Mechanism of Double-Strand DNA Break Repair by the Nonhomologous DNA EndJoining Pathway. Annual review of biochemistry 2010, 79, 181-211, doi:10.1146/annurev.biochem.052308.093131.

7. Chang, H.H.Y.; Pannunzio, N.R.; Adachi, N.; Lieber, M.R. Non-Homologous DNA End Joining and Alternative Pathways to Double-Strand Break Repair. Nature reviews. Molecular cell biology 2017, 18, 495-506, doi:10.1038/nrm.2017.48.

8. Gutschner, T.; Haemmerle, M.; Genovese, G.; Draetta, G.F.; Chin, L. Post-Translational Regulation of Cas9 during G1 Enhances Homology-Directed Repair. Cell reports 2016, 14, 1555-1566, doi:10.1016/j.celrep.2016.01.019.

9. Wilbie, D.; Walther, J.; Mastrobattista, E. Delivery Aspects of CRISPR/Cas for in Vivo Genome Editing. Accounts of Chemical Research 2019, 52, 1555-1564, doi:10.1021/acs.accounts.9b00106.

10. Legut, M.; Dolton, G.; Mian, A.A.; Ottmann, O.G.; Sewell, A.K. CRISPR-Mediated TCR Replacement Generates Superior Anticancer Transgenic T Cells. Blood 2018, 131, 311-322, doi:10.1182/blood-2017-05-787598.

11. Stadtmauer, E.A.; Fraietta, J.A.; Davis, M.M.; Cohen, A.D.; Weber, K.L.; Lancaster, E.; Mangan, P.A.; Kulikovskaya, I.; Gupta, M.; Chen, F.; et al. CRISPR-Engineered T Cells in Patients with Refractory Cancer. Science (New York, N.Y.) 2020, 367, doi:10.1126/science.aba7365.

12. Lu, Y.; Xue, J.; Deng, T.; Zhou, X.; Yu, K.; Deng, L.; Huang, M.; Yi, X.; Liang, M.; Wang, Y.; et al. Safety and Feasibility of CRISPR-Edited T Cells in Patients with Refractory Non-Small-Cell Lung Cancer. Nature medicine 2020, 26, 732-740, doi:10.1038/s41591-020-0840-5.

13. Finn, J.D.; Smith, A.R.; Patel, M.C.; Shaw, L.; Youniss, M.R.; van Heteren, J.; Dirstine, T.; Ciullo, C.; Lescarbeau, R.; Seitzer, J.; et al. A Single Administration of CRISPR/Cas9 Lipid Nanoparticles Achieves Robust and Persistent In Vivo Genome Editing. Cell Reports 2018, 22, 2455-2468, doi:10.1016/j.celrep.2018.02.014.

14. Ledford, H. CRISPR Treatment Inserted Directly into the Body for First Time. Nature 2020, $579,185$.

15. Suzuki, Y.; Onuma, H.; Sato, R.; Sato, Y.; Hashiba, A.; Maeki, M.; Tokeshi, M.; Kayesh, M.E.H.; Kohara, M.; Tsukiyama-Kohara, K.; et al. Lipid Nanoparticles Loaded with Ribonucleoprotein-Oligonucleotide Complexes Synthesized Using a Microfluidic Device Exhibit Robust Genome Editing and Hepatitis B Virus Inhibition. Journal of controlled release: official journal of the Controlled Release Society 2021, 330, 61-71, doi:10.1016/j.jconrel.2020.12.013. 
16. Li, Y.; Bolinger, J.; Yu, Y.; Glass, Z.; Shi, N.; Yang, L.; Wang, M.; Xu, Q. Intracellular Delivery and Biodistribution Study of CRISPR/Cas9 Ribonucleoprotein Loaded Bioreducible Lipidoid Nanoparticles. Biomaterials science 2019, 7, 596-606, doi:10.1039/c8bm00637g.

17. Patel, S.; Ashwanikumar, N.; Robinson, E.; Duross, A.; Sun, C.; Murphy-Benenato, K.E.; Mihai, C.; Almarsson, Ö.; Sahay, G. Boosting Intracellular Delivery of Lipid Nanoparticle-Encapsulated MRNA. Nano Letters 2017, 17, 5711-5718, doi:10.1021/acs.nanolett.7b02664.

18. Kouranova, E.; Forbes, K.; Zhao, G.; Warren, J.; Bartels, A.; Wu, Y.; Cui, X. CRISPRs for Optimal Targeting: Delivery of CRISPR Components as DNA, RNA, and Protein into Cultured Cells and Single-Cell Embryos. Human Gene Therapy 2016, 27, 464-475, doi:10.1089/hum.2016.009.

19. Ma, H.; Tu, L.-C.; Naseri, A.; Huisman, M.; Zhang, S.; Grunwald, D.; Pederson, T. CRISPR-Cas9 Nuclear Dynamics and Target Recognition in Living Cells. The Journal of cell biology 2016, 214, 529-537, doi:10.1083/jcb.201604115.

20. Tu, Z.; Yang, W.; Yan, S.; Yin, A.; Gao, J.; Liu, X.; Zheng, Y.; Zheng, J.; Li, Z.; Yang, S.; et al. Promoting Cas9 Degradation Reduces Mosaic Mutations in Non-Human Primate Embryos. Scientific reports 2017, 7, 42081, doi:10.1038/srep42081.

21. Lin, S.; Staahl, B.T.; Alla, R.K.; Doudna, J.A. Enhanced Homology-Directed Human Genome Engineering by Controlled Timing of CRISPR/Cas9 Delivery. eLife 2014, 3, e04766, doi:10.7554/eLife.04766.

22. Klein, M.; Eslami-Mossallam, B.; Arroyo, D.G.; Depken, M. Hybridization Kinetics Explains CRISPR-Cas OffTargeting Rules. Cell reports 2018, 22, 1413-1423, doi:10.1016/j.celrep.2018.01.045.

23. Wei, T.; Cheng, Q.; Min, Y.L.; Olson, E.N.; Siegwart, D.J. Systemic Nanoparticle Delivery of CRISPR-Cas9 Ribonucleoproteins for Effective Tissue Specific Genome Editing. Nature Communications 2020, 11, 1-12, doi:10.1038/s41467-020-17029-3.

24. Manning, M.C.; Chou, D.K.; Murphy, B.M.; Payne, R.W.; Katayama, D.S. Expert Review Stability of Protein Pharmaceuticals : An Update. 2010, 27, 544-575, doi:10.1007/s11095-009-0045-6.

25. Love, K.T.; Mahon, K.P.; Levins, C.G.; Whitehead, K.A.; Querbes, W.; Dorkin, J.R.; Qin, J.; Cantley, W.; Qin, L.L.; Racie, T.; et al. Lipid-like Materials for Low-Dose, in Vivo Gene Silencing. Proceedings of the National Academy of Sciences 2010, 107, 1864 LP - 1869, doi:10.1073/pnas.0910603106.

26. D'Astolfo, D.S.; Pagliero, R.J.; Pras, A.; Karthaus, W.R.; Clevers, H.; Prasad, V.; Lebbink, R.J.; Rehmann, H.; Geijsen, N. Efficient Intracellular Delivery of Native Proteins. Cell 2015, 161, 674-690, doi:10.1016/j.cell.2015.03.028.

27. Alonso Villela, S.M.; Kraïem, H.; Bouhaouala-Zahar, B.; Bideaux, C.; Aceves Lara, C.A.; Fillaudeau, L. A Protocol for Recombinant Protein Quantification by Densitometry. MicrobiologyOpen 2020, 9, 1175-1182, doi:10.1002/mbo3.1027.

28. Burger, A.; Lindsay, H.; Felker, A.; Hess, C.; Anders, C.; Chiavacci, E.; Zaugg, J.; Weber, L.M.; Catena, R.; Jinek, M.; et al. Maximizing Mutagenesis with Solubilized CRISPR-Cas9 Ribonucleoprotein Complexes. Development (Cambridge, England) 2016, 143, 2025-2037, doi:10.1242/dev.134809.

29. de Jong, O.G.; Murphy, D.E.; Mäger, I.; Willms, E.; Garcia-Guerra, A.; Gitz-Francois, J.J.; Lefferts, J.; Gupta, D.; Steenbeek, S.C.; van Rheenen, J.; et al. A CRISPR-Cas9-Based Reporter System for Single-Cell Detection of Extracellular Vesicle-Mediated Functional Transfer of RNA. Nature Communications 2020, 11, 1113, doi:10.1038/s41467-020-14977-8.

30. Glaser, A.; McColl, B.; Vadolas, J. GFP to BFP Conversion: A Versatile Assay for the Quantification of CRISPR/Cas9-Mediated Genome Editing. Molecular therapy. Nucleic acids 2016, 5, e334, doi:10.1038/mtna.2016.48. 
31. Hu, Y.; Crist, R.M.; Clogston, J.D. The Utility of Asymmetric Flow Field-Flow Fractionation for Preclinical Characterization of Nanomedicines. Analytical and bioanalytical chemistry 2020, 412, 425-438, doi:10.1007/s00216-019-02252-9.

32. Liu, Y.; Fens, M.H.A.M.; Capomaccio, R.B.; Mehn, D.; Scrivano, L.; Kok, R.J.; Oliveira, S.; Hennink, W.E.; van Nostrum, C.F. Correlation between in Vitro Stability and Pharmacokinetics of Poly( $\varepsilon$-Caprolactone)-Based Micelles Loaded with a Photosensitizer. Journal of controlled release : official journal of the Controlled Release Society 2020, 328, 942-951, doi:10.1016/j.jconrel.2020.10.040.

33. Caputo, F.; Arnould, A.; Bacia, M.; Ling, W.L.; Rustique, E.; Texier, I.; Mello, A.P.; Couffin, A.-C. Measuring Particle Size Distribution by Asymmetric Flow Field Flow Fractionation: A Powerful Method for the Preclinical Characterization of Lipid-Based Nanoparticles. Molecular pharmaceutics 2019, 16, 756-767, doi:10.1021/acs.molpharmaceut.8b01033.

34. Akinc, A.; Maier, M.A.; Manoharan, M.; Fitzgerald, K.; Jayaraman, M.; Barros, S.; Ansell, S.; Du, X.; Hope, M.J.; Madden, T.D.; et al. The Onpattro Story and the Clinical Translation of Nanomedicines Containing Nucleic AcidBased Drugs. Nature Nanotechnology 2019, 14, 1084-1087, doi:10.1038/s41565-019-0591-y.

35. Guimaraes, P.P.G.; Zhang, R.; Spektor, R.; Tan, M.; Chung, A.; Billingsley, M.M.; El-Mayta, R.; Riley, R.S.; Wang, L.; Wilson, J.M.; et al. Ionizable Lipid Nanoparticles Encapsulating Barcoded MRNA for Accelerated in Vivo Delivery Screening. Journal of Controlled Release 2019, 316, 404-417, doi:10.1016/j.jconrel.2019.10.028.

36. Sebastiani, F.; Yanez Arteta, M.; Lerche, M.; Porcar, L.; Lang, C.; Bragg, R.A.; Elmore, C.S.; Krishnamurthy, V.R.; Russell, R.A.; Darwish, T.; et al. Apolipoprotein E Binding Drives Structural and Compositional Rearrangement of MRNA-Containing Lipid Nanoparticles. ACS Nano 2021, 15, 6709-6722, doi:10.1021/acsnano.0c10064.

37. Nguyen, D.N.; Roth, T.L.; Li, P.J.; Chen, P.A.; Apathy, R.; Mamedov, M.R.; Vo, L.T.; Tobin, V.R.; Goodman, D.; Shifrut, E.; et al. Polymer-Stabilized Cas9 Nanoparticles and Modified Repair Templates Increase Genome Editing Efficiency. Nature biotechnology 2020, 38, 44-49, doi:10.1038/s41587-019-0325-6. 